\title{
Chanter les poètes avec (ou sans) les maths
}

\author{
Moreno ANDREATTA (Strasbourg) ${ }^{1}$
}

\section{Summary}

Starting from a personal experience in turning texts by poets into songs, I will show how mathematics might shed some new light on our understanding of the relation between poetry and music. After recalling the conflictual relations between poetry and chanson, I will focus on a geometric representation of the harmonic space - the Tonnetz - that enabled me to propose an original perspective on poetic song writing through the concept of 'Hamiltonian song'. Two musical examples will illustrate the flexibility of this formal approach in poetry-based song writing.

\section{Introduction}

La hiérarchisation de la poésie et de la chanson n'est pas appropriée, car d'une part l'une n'est pas l'autre, et d'autre part aucune caractéristique ne la justifie. Il est de bon ton parfois de railler la simplicité d'une chanson, à côté d'un poème qui, forcément, n'aurait pas cette simplicité. Si la simplicité signifie l'intelligibilité, il n'y a pas que des chansons qui la possèdent. S'il s'agit du simplisme, alors c'est un défaut, qui n'est propre ni à la poésie ni à la chanson en tant que genres, mais qui peut se retrouver dans l'une comme dans l'autre. Si la simplicité consiste à exprimer des sentiments avec des mots simples, alors la poésie comme la chanson peut le faire. (Vincenot 2017, 114)

Dans l'histoire millénaire des rapports entre musique et littérature, des Grecs à nos jours, la mise en chanson de textes poétiques constitue un véritable objet de fascination pour les musicologues. Les rapports entre la chanson et la poésie ont été souvent conflictuels, une sorte de "cousinage compliqué ", pour reprendre une belle formule employée par le poète Matthias Vincenot dans un ouvrage auquel on fera souvent référence au cours de ce travail (Vincenot 2014). Cependant, au-delà de nombreux malentendus qui, comme on le verra, ont marqué le débat sur la relation entre poésie et chanson, force est de constater que les approches musicologiques contemporaines, plus ouvertes au domaine de la popular music, ont su changer la donne et imposer un regard attentif sur cette modalité d'existence de l'objet ‘ chanson `. Plutôt que s'intéresser au côté littéraire et, plus spécifiquement poétique, de certaines chansons, et en particulier de celles dites ‘ à texte ', on peut focaliser l'attention sur 
un phénomène qui semble avoir quitté le cercle des spécialistes pour envahir la sphère du grand public. Doit-on continuer à considérer la poésie mise en chanson comme une niche au sein de la catégorie des chansons à texte ou bien assiste-t-on à un véritable engouement de la part du marché musical susceptible de transformer cette exception culturelle en un véritable canon ?2 Pour cela il conviendra tout d'abord de revenir brièvement sur quelques aspects de ce ' malentendu > concernant les rapports entre chanson et poésie, en essayant d'analyser ce phénomène dans une perspective historique dépassant le cadre de la chanson française à textes mais incluant également la canzone d'autore italienne, qui est sans doute celle ayant tissé le plus de liens avec la poésie française mise en chanson. ${ }^{3}$

\section{Les malentendus du rapport entre poésie et chanson}

La question du rapport conflictuel entre poésie et chanson ainsi que de certains malentendus qui ont accompagné la naissance de la chanson à textes en France et de la chanson d'auteur en Italie a fait l'objet de nombreuses études. La question est traitée en détail par Céline Pruvost dans la deuxième partie de sa thèse de doctorat consacrée aux spécificités méthodologiques de l'étude de chanson (Pruvost 2013), étude à laquelle nous renvoyons le lecteur. ${ }^{4} \mathrm{Au}$-delà des différences culturelles entre chanson à textes en France et chanson d'auteur en Italie, il n'y a pas de doute sur le consensus quant à l'existence d'un rapport conflictuel entre ces deux expressions artistiques, la poésie et la chanson, dont le cousinage compliqué touche aux différences profondes entre les deux formes d'activités artistiques. Matthias Vincenot nous met en garde face à la tentation d'établir des rapports directs et simplistes entre poésie et chanson :

Que la chanson soit dite poétique ou non, que la poésie chante ou non, que celleci s'adresse au plus grand nombre ou pas, la poésie n'est pas la chanson dans l'écriture-même. La langue poétique vise la suggestion, elle laisse imaginer. [...] La chanson, elle, dépend d'une régularité de forme liée au tempo musical. Si l'auteur décide de bousculer l'ordre imposé par la musique, il faudra que la musique puisse le rendre, et si elle ne le peut pas, ce bousculement sera plus difficile. Dans l'écriture d'une chanson, c'est donc la musique qui va déclencher le rythme. (Vincenot 2014, 198)

En effet, " un poème, même s'il peut être mis en musique et chanté, n'est pas, au départ, une chanson, puisqu'il n'a pas été écrit avec l'idée d'une musique et d'une interprétation " (Vincenot 2014, 25). C'est un constat qui s'accorde parfaitement avec la réflexion de Georges Moustaki sur celle qu'il appelle la recette d'une chanson réussie :

Le propre d'une chanson réussie est que les paroles et la musique ne peuvent se suffire à elles-mêmes et qu'il y a entre elles une dépendance indéfectible. [...] Sans qu'il y ait une différence rigide, je peux dire confusément que le choix des mots n'est pas le même quand on sait que le texte sera lu, récité ou chanté. Peut-être qu’un poème s'adresse à 
soi-même tandis qu'une chanson a pour vocation de s'adresser au plus grand nombre. On le voit, je tourne autour du pot sans réussir à donner une réponse catégorique aux demandeurs de la recette [d'une chanson réussie]..$^{5}$ (Moustaki 2011, 23-24)

Bob Dylan, prix Nobel de littérature pour " avoir créé de nouvelles expressions poétiques, dans le sillage de la chanson traditionnelle américaine " ${ }^{6}$ exprimera un point de vue très proche lors de sa réponse, sous la forme d'un enregistrement audio, à l'Académie royale des sciences de Suède, des propos que je vais reprendre ici directement dans la traduction française parue ensuite chez Fayard :

Nos chansons sont vivantes au pays des vivants. Mais contrairement à la littérature, les chansons sont destinées à être chantées, et non pas lues. Les textes des pièces de Shakespeare avaient pour vocation d'être dits sur scène. De même, les paroles des chansons ont pour vocation d'être chantées et non pas lues sur une page. (Dylan 2017, 32-33)

Cependant, si nul ne doute que la chanson ne soit une branche de la poésie, pas plus que la poésie ne soit une branche de la chanson ${ }^{7}$,

[1]a poésie chantée est, elle, une branche de la chanson, parce qu'elle est une façon, chantée justement, de s'approprier la poésie, selon que le compositeur décide de chanter le poème tel qu'il est écrit (d'une façon qui n'est pas initialement prévue pour être chantée), ou qu'il opère quelques changements de forme pour lui donner une forme traditionnelle de chanson, avec des couplets et un refrain. (Vincenot 2014, 425)

L'existence même de la poésie chantée en tant que catégorie a sans doute contribué à alimenter le malentendu entre poésie et chanson, comme l'atteste le célèbre échange entre les trois ‘ poètes > de la chanson à textes française, Brel, Brassens et Ferré. Au-delà de l'attribution ou pas du statut d'art majeur à la chanson, les trois musiciens s'accordent sur le fait que leur pratique consiste à " mélanger des paroles et de la musique ", pour reprendre l'expression employée par Brassens, plutôt qu'à faire de la poésie, celle-ci étant faite pour être lue ou dite à la différence de la chanson qui demande à " employer un vocabulaire un peu différent, des mots qui accrochent l'oreille plus vite [...] " ${ }^{8}$. Le fait de mélanger des paroles et de la musique, comme Brassens le dit clairement face à ces deux interlocuteurs, est en effet

tout à fait différent de ce qu'on appelle couramment la poésie, qui est faite pour être lue ou dite. La chanson c'est très différent. Même si des types comme Ferré ont réussi à mettre des poètes en musique, comme Baudelaire, il est difficile d'utiliser la chanson comme les poètes qui nous ont précédés utilisaient le verbe. Quand on écrit pour l'oreille, on est quand même obligé d'employer un vocabulaire un peu différent, des mots qui accrochent l'oreille plus vite [...] Bien qu'on l'ait aussi avec le disque, le lecteur a plus facilement la possibilité de revenir en arrière $[\ldots] .^{9}$ 


\section{Mise en musique et mise en chanson de textes poétiques}

Si la mise en chanson de textes poétiques constitue, ou pourra constituer, un véritable canon au sein des différents genres musicaux, il convient de ne pas confondre cette pratique avec une démarche qui est, quant à elle, beaucoup plus ancienne, à savoir celle de la mise en musique de poèmes. ${ }^{10}$ Comme l'explique clairement Stéphane Hirschi à partir de sa démarche cantologique dans l'étude de l'objet ‘ chanson ? :

Il s'agit d'abord de distinguer mise en chanson et mise en musique. La mise en musique repose sur un respect du texte premier. [...] La chanson introduit, insère dans l'œuvre, outre une ligne mélodique, une nouvelle dimension, une temporalité donnée, fixée par l'interprétation enregistrée sur une mesure. [...] La mise en chanson [...] insère donc un devenir, une temporalité sensible, celle de la mesure, par-delà l'inscription dans l'espace dessinée par l'écriture poétique. Dans cette logique d'audition sans retour possible en arrière, la structure musicale a pour fonction d'insuffler un dynamisme aux évocations textuelles. [...] On conçoit donc que toute mise en musique d'un poème ne determine pas nécessairement sa mise en chanson: sans la présence sensible d'un air fredonnable, d'une pulsation et de formes de répétitions destinées à dilater la matière précieuse de cet air compté, on aboutit soit au genre aristocratique de la mélodie, illustrée par Fauré et Debussy, soit au poème chanté, comme lorsque Ferré met ses notes au service de Verlaine ou Rimbaud : [...] le texte y éclipse l'ensemble. (Hirschi 2008, 70)

Bien différent est le cas de la mise en chanson, toujours de la part de Ferré, des poèmes de Baudelaire ou d'Aragon, une activité que le musicien a souvent décrite en soulignant le rôle primordial de l'intuition. En effet :

Tous les poèmes de Baudelaire, je les ai mis en musique à l'instinct. Je mets les paroles sur mon piano et j'improvise immédiatement. [...] Il faut improviser, il faut que ça vienne sur le clavier, il faut que les mains parlent en même temps que les yeux lisent la poésie. (Pistone 2018, 35) 11 $^{11}$

Cette relation instinctive que le compositeur entretient avec le texte poétique ne concerne bien évidemment pas uniquement Baudelaire. C'est le cas par exemple de la rencontre avec les textes d'Aragon, poète que Ferré affirme avoir rencontré

[...] au cœur même de ses mots. Je l'ai lu avec mes mains enchaînées au clavier et à ma voix. Entendons-nous bien : cela n'est pas une formule, ni une image, mais l'expression d'une technique. Le vers d'Aragon est, en dehors de toute évocation, branché sur la musique. On a pris l'habitude d'écrire, dans les manuels de littérature, que le vers se suffit à lui-même et que les syllabes chantent, que la rime ou l'assonance accusent les contours de la mélodie verbale. (Ferré 1961) 
De même que pour les poèmes de Baudelaire : « Derrière la porte des paroles d'Aragon, il y avait une musique que j'ai trouvée, immédiatement. Et quand cela n'était pas immédiat, je tournais la page et passais à d'autres portes. J'ai mis Aragon en musique de la même façon que j'ai mis en musique Rutebeuf. » (Ferré 1961)

L'immédiateté et la composante instinctive de la mise en chanson d'un texte poétique n'est cependant pas synonyme de subjectivité absolue. En effet «La musique pure est subjective. La musique, épouse d'un texte, par contre, est objective. Le mariage est bon ou il n'est pas. Il n'y a pas de faux couple, pas en tout cas qui relève de la critique. Ce mariage-là est un don du hasard, de la rencontre. » (Ferré 1961)

Il s'agit, comme Ferré l'explicite clairement dans le même texte, de la rencontre entre le verbe et la mélodie, car en réalité :

[...] ce qu'Aragon déploie dans la phrase poétique n'a besoin d'aucun support [...] mais la matière même de son langage est faite pour la mise sur le métier des sons. Je ne crois pas à la collaboration, mais à une double vue, celle du poète qui a écrit, celle du musicien qui voit ensuite, et qui perçoit des images musicales derrière la porte des paroles. (Ferré 1961)

Il n'est donc pas tout à fait étonnant qu'Aragon puisse avoir vu en Leo Ferré un poète plus qu'un chansonnier, à savoir quelqu'un qui :

[...] écrit directement ses poèmes suivant les lois d'un genre poétique, la chanson. Là est la raison de ce don singulier qu'il a de récrire les poèmes des autres, de pratiquer un art très singulier qu'il faut bien appeler la mise en chanson des poèmes. Il ne l'a point inventé, mais il l'a poussé parfois à un degré de perfection dont témoigne la vie rendue à un poème de Rutebeuf, mise en chanson exemplaire qui est comme une magistrale restauration de tableau et promet, si cet exemple est suivi, la restitution à l'humanité de demain de milliers de trésors enfouis sous les bitumes de l'ancien langage. (Aragon 1961)

La mise en chanson d'un poème devient ainsi, aux yeux d'Aragon, « une forme supérieure de la critique poétique ». Il s'agit d'une véritable " critique créatrice ", car " elle recrée le poème, elle y choisit, elle donne à un vers une importance, une valeur qu'il n'avait pas, le répète, en fait un refrain... ». Le musicien peut ainsi rendre "à la poésie un service dont on calcule mal encore la portée, en mettant à la disposition du nouveau lecteur, un lecteur d'oreille, la poésie doublée de la magie musicale (Aragon 1961). 
Stratégies formelles de mise en chanson d'un texte poétique : le Tonnetz et le concept de ' chanson hamiltonienne ?

Face à la tâche délicate de faire chanter un texte poétique, au sens de transformer une poésie en chanson, j'ai personnellement choisi une voie très différente de celle purement intuitive qui semble caractériser l'acte poético-compositionnel de Léo Ferré. Sans négliger le rôle ô combien nécessaire de l'intuition (soit-elle pure ou arithmétique, comme Pascal Pistone définit la démarche singulière de Léo Ferré), je me suis tourné plutôt vers la formalisation géométrique afin de pouvoir explorer des techniques de modulations que l'on trouve rarement dans la chanson. En particulier, il s'agit de trouver des stratégies simples pour moduler dans toutes les tonalités tout en respectant les caractéristiques de simplicité formelle du genre chanson à savoir une relative brièveté, la présence d'éléments aisément mémorisables (les hooks ou < crochets `) à la fois dans la ligne mélodique et dans la structure harmonique ainsi que de segments reconnaissables (tels le couplet, le refrain, le pont...) ${ }^{12}$. Pour ce faire, on peut s'appuyer sur des représentations géométriques dont les origines remontent à la deuxième moitié du XVIII siècle. Il s'agit du Tonnetz (ou « réseau des notes `), une représentation initialement conçue par le mathématicien et théoricien de la musique suisse Leonard Euler et qui a connu un regain d'intérêt dans les vingt dernières années en particulier au sein de la tradition analytique américaine. Prenant inspiration également des travaux du musicologue Hugo Riemann, l’approche dite ‘ néo-riemannienne > s’appuie sur le Tonnetz en tant que support d'analyse harmonique. Il s'agit d'une triangulation de l'espace bidimensionnel ou chaque triangle représente soit un accord majeur soit un accord mineur, les deux types d'accords étant reliés par trois symétries. Ces transformations s'appellent respectivement le « parallèle > (opérateur indiqué par P), le 〈relatif) (R) et le (Leittonwechsel) (L). Elles sont déployées dans une portion du Tonnetz et représentées en Figure 1.

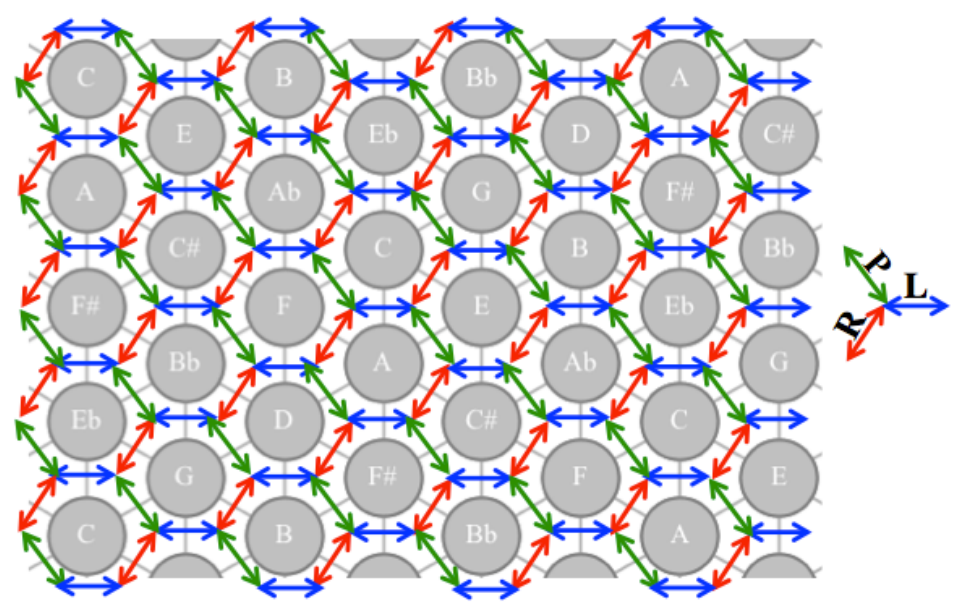

Figure 1. Les trois transformations néo-riemanniennes ( $\mathrm{P}$ ou ‘ parallèle , $\mathrm{R}$ ou ‘ relatif > et $\mathrm{L}$ ou

(Leittonwechsel ,) permettant de passer d'une triade majeure à une triade mineure ayant avec l'accord de départ deux notes en commun 
En utilisant cet espace géométrique pour décrire des progressions harmoniques il est possible d'étudier des trajectoires spatiales correspondants à des modulations permettant de passer à travers toutes les tonalités majeures et mineures sans répéter deux fois le même accord et en revenant au point de départ. Un tel parcours harmonique correspond à ce qu'on appelle en théorie des graphes un ' cycle hamiltonien ', ce qui permet de passer de façon « lisse ` d'un accord à l'accord successif en gardant deux notes en commun. Ce procédé m’a emmené à définir le concept de ‘ chanson hamiltonienne s en tant que sous-genre de la chanson poétique déployant une complexité maximale dans la structure harmonique, ce qui représente un véritable défi compte-tenu des contraintes formelles du genre chanson que nous avons rappelées précédemment. De plus, selon le type de symétrie à la base du cycle on peut jouer sur la boucle rétention/protension en maintenant l'auditeur dans une attente constante de l'accord qui va suivre, accord qui ne sera jamais perçu comme totalement dissocié du contexte harmonique précisément à cause de la présence de deux notes en commun entre n'importe quel accord et celui qui suivra. Autrement dit, chaque accord est lié à l'accord suivant par ce qu'on appelle en jargon musical une < conduite parcimonieuse des voix > (minimal voice leading). J'ai employé ce procédé dans la mise en chanson du poème "Aprile " du poète italien de la Décadence, Gabriele d'Annunzio en alternant les couplets avec des parties instrumentales correspondant à trois cycles hamiltoniens différents mais ayant les mêmes caractéristiques structurelles. Les trois cycles ne peuvent en effet pas être décomposés en sous-cycles, ce qui leur donne une sorte de complexité maximale par rapport à d'autres cycles hamiltoniens dits $\triangleleft$ redondants $\iota^{13}$.

$$
\begin{gathered}
\text { "Aprile » } \\
\text { (d'après Gabriele D’Annunzio) }
\end{gathered}
$$

Socchiusa è la finestra, sul giardino. Un'ora passa lenta, sonnolenta.

Ed ella, ch'era attenta, s'addormenta a quella voce che già si lamenta, - che si lamenta in fondo a quel giardino.
La fenêtre est entr'ouverte, sur le jardin.

Une heure passe, lente, somnolente.

Et elle, d'abord attentive, finit par s'endormir

A cette voix qui là-bas se lamente,

Qui se lamente au fond de ce jardin.

[Partie instrumentale avec premier cycle hamiltonien]

Non è che voce d'acque su la pietra: e quante volte, quante volte udita! Quell'amore e quell'ora in quella vita saffondan come ne l'onda infinita stretti insieme il cadavere e la pietra.
Ce n'est qu'une voix d'eau sur la pierre, Et combien de fois, combien de fois entendue !

Cet amour et cette heure s'abîment dans cette vie Comme s'abîment dans l'onde sans fin Le cadavre et la pierre liés ensemble.

[Partie instrumentale avec deuxième cycle hamiltonien] 
Ella stende l'angoscia sua nel sonno.

L'angoscia è forte, e il sonno è così lieve!

(Par la luce d'april quasi una neve

che sia tiepida.)

Ed ella certo deve soffrire,

vagamente, anche nel sonno.
Elle détend son angoisse dans le sommeil.

Mais l'angoisse est forte, et le sommeil est si léger !

(La lumière d'avril ressemble presque à une neige qui serait tiède.)

Et certes elle doit souffrir,

Vaguement souffrir, aussi dans le sommeil.

[Partie instrumentale avec troisième cycle hamiltonien]

La figure suivante (Figure 2) offre une représentation des trois cycles précédents qui correspondent aux trois trajectoires distinctes dans le Tonnetz. Le premier cycle utilisé dans la chanson est représenté également en notation musicale traditionnelle.
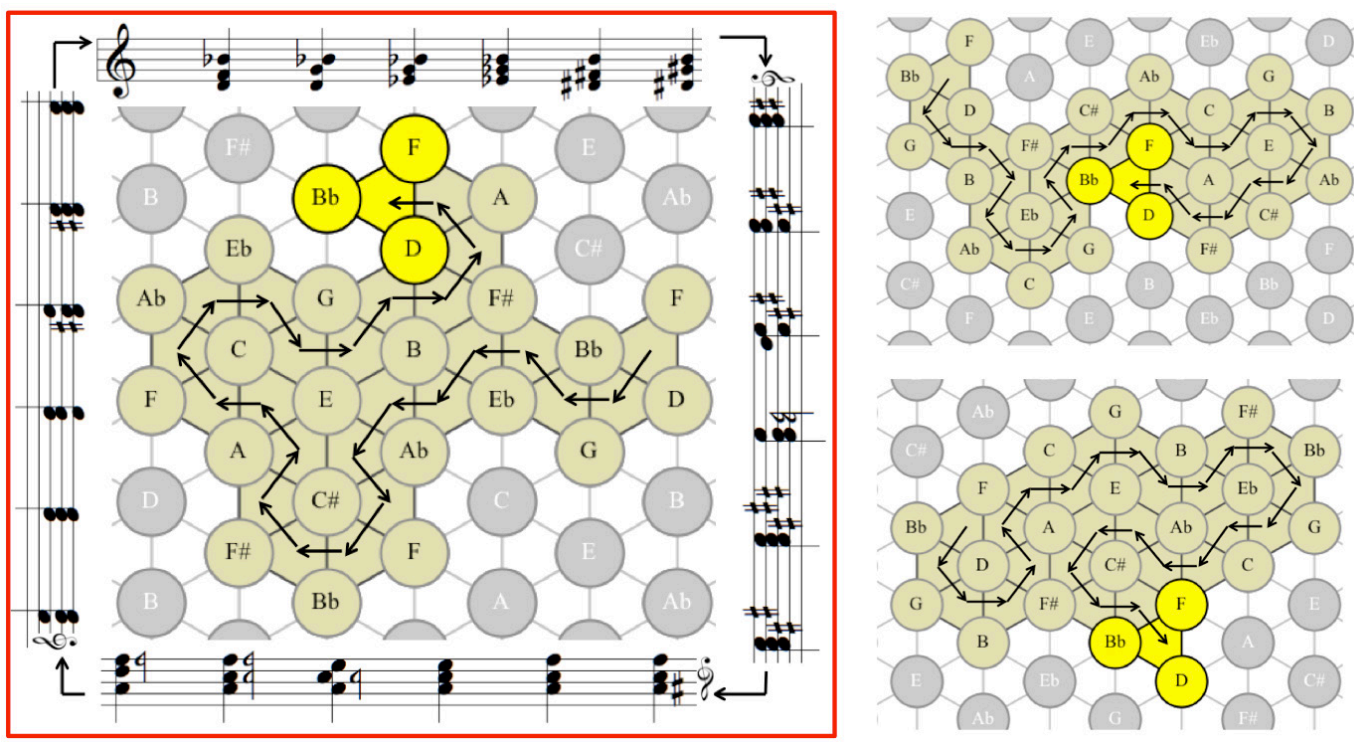

Figure 2. Les trois cycles hamiltoniens structurellement différents utilisés dans la partie instrumentale de la chanson «Aprile » composée à partir d'un poème de Gabriele D’Annunzio

Dans une expérience successive, j'ai essayé de réduire la complexité du caractère hamiltonien du procédé à travers des cycles ayant des périodicités internes. Le nouveau défi a été celui d'appliquer une telle progression harmonique non seulement dans la partie instrumentale mais dans le texte de la chanson, ce qui comporte la création d'une mélodie respectant à la fois la scansion rythmique du texte et également la structure harmonique du cycle hamiltonien sous-jacent. Le cycle est donné en figure 3 et correspond, comme on peut le voir assez clairement, à une trajectoire spatiale qui - à la différence des cycles précédents - peut se décomposer en sous-parties (plus exactement en quatre cycles identiques de longueur six). 


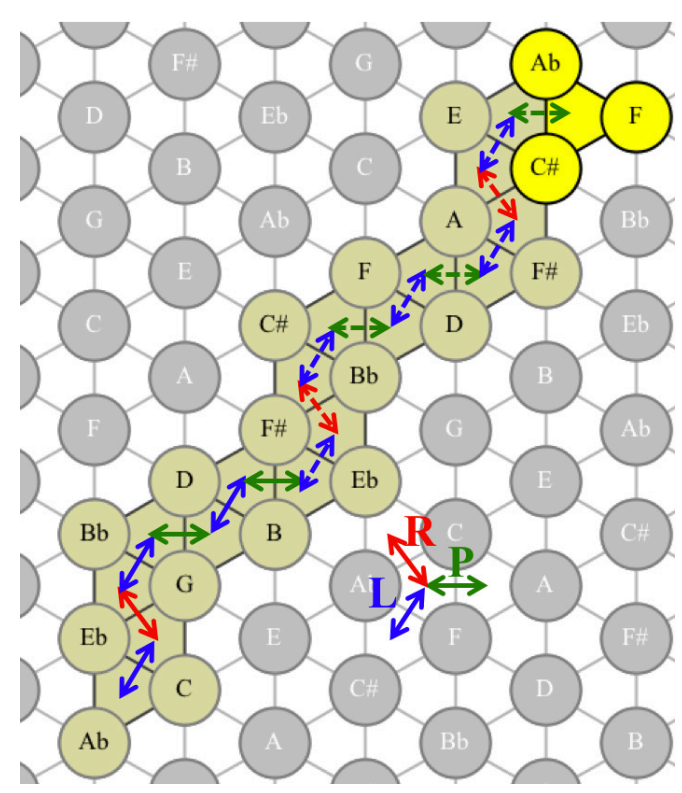

Figure 3. Cycle hamiltonien utilisé dans la chanson "La sera non è più la tua canzone " composée à partir d'un poème de Mario Luzi

Le code couleur utilisé pour indiquer les trois opérateurs néo-riemanniens (rouge pour le relatif $\mathrm{R}$, vert pour le parallèle $\mathrm{P}$ et bleu pour le Leittonwechsel $\mathrm{L}$ ) devrait permettre au lecteur de comprendre le travail nécessaire pour trouver une mélodie s'adaptant à la fois au texte poétique mais aussi à la progression harmonique parcourant les vingt-quatre accords majeurs et mineurs à partir de la séquence LRLPLP (répétée quatre fois, histoire d'arriver à une séquence susceptibles de parcourir l'espace des vingt-quatre accords majeurs et mineurs). ${ }^{14}$

"La sera non è più la tua canzone "

(d'après Mario Luzi. Traduction d'Antonia Soulez et Alexandre Schmitt)

La sera non è più la tua canzone, è questa roccia d'ombra traforata dai lumi e dalle voci senza fine, la quiete d'una cosa già pensata.

Ah questa luce viva e chiara viene solo da te, sei tu cosi vicina al vero d'una cosa conosciuta, per nome hai una parola ch'e passata nell'intimo del cuore e s'è perduta.
Le soir n'est plus ta chanson

C'est ce rocher d'ombre transpercé

Par les lumières et les voix sans fin,

La paix d'une chose déjà pensée.

$\mathrm{Ah}$, cette lumière vive et claire émane

De toi uniquement, tu es si proche

De la réalité d'une chose connue,

Tu as pour nom une parole passée

Par l'intimité du cœur avant de s'y perdre. 
Caduto è più che un segno della vita, riposi, dal viaggio sei tornata dentro di te, sei scesa in questa pura sostanza cosi tua, cosi romita

nel silenzio dell'essere, nel silenzio dell'essere.

L'aria tace ed il tempo dietro a te si leva come un'arida montagna dove vaga il tuo spirito e si perde, un vento raro scivola e ristagna.

Sei scesa in questa pura

sostanza cosi tua

cosi romita

nel silenzio dell'essere, compiuta
Déchu il est plus qu'un signe de vie,

Reposes, du voyage tu es revenue

Tu es descendue en toi, dans cette pure substance qui est si tienne,

Si éloignée

Dans le silence de l'être

Dans le silence de l'être,

L'air se tait et le temps derrière toi

Se lève tel une montagne aride

Où ton esprit plane puis il se perd,

Un vent inhabituel souffle puis s'estompe.

Tu es descendue dans cette

Pure substance qui est si tienne,

Si éloignée

Dans le silence de l'être,

Accomplie dans le silence de l'être.

\section{Conclusion : la poésie mise en chanson a-t-elle besoin des mathématiques ?}

Si c'est vrai, comme l'a bien souligné Véronique Pestel à propos des poèmes d'Aragon, que " faire une chanson à partir de l'un d'eux suppose un point de vue [car] c'est le compositeur qui définit le territoire, choisit les strophes, impose le rythme, revisite la géographie et réinvente l'histoire " (Pestel 2012, 81), l'utilisation des mathématiques n'est finalement qu'une des possibles stratégies pour explorer des nouvelles formes de dialogue entre un texte poétique et sa mise en chanson. En particulier, le recours à des cycles hamiltoniens sur le Tonnetz comme architecture harmonique d'une chanson ouvre, à nos yeux, des perspectives inédites pour complexifier la structure formelle du genre chanson sans renoncer à sa spécificité qui est avant tout celle d'être un " air fixé par des paroles" (Hirschi 2008, 29) touchant à la dimension intime de l'existence. Car, comme bien l'affirme Matthias Vincenot en conclusion de son analyse de ce cousinage compliqué entre poésie et chanson, ce qui les rapproche c'est « ce qu'elles suscitent : un rapport intime qu'on peut avoir avec tel ou tel vers, tel ou tel refrain. C'est aussi leur vocation : parler à tous, et/ou à chacun, d'eux-mêmes, de soi-même. En effet, si la poésie et la chanson, chacune à sa manière, parlent de ce qui nous entoure, elles parlent, d'abord et avant tout, de nous. » (Vincenot 2014, 430) 


\section{Notes}

1 Moreno Andreatta est directeur de recherche CNRS en mathématiques et musique à l'IRMA / Université de Strasbourg, membre du GREAM (Groupe de Recherches Expérimentales sur l'Acte Musical) et chercheur associé à l'équipe Représentations musicales de l'Ircam.

2 L'existence d'une telle catégorie au sein de la popular music était, en quelque sorte, l'une des principales hypothèses avancées lors du colloque Chanter les poètes (Université Grenoble Alpes, 16-18 octobre 2018). Je remercie les organisatrices, Caroline Bertonèche et Anne Cayuela, pour l'invitation à participer à ce colloque ainsi que pour les discussions très stimulantes qui ont accompagné les trois journées et qui m’ont permis de clarifier les fondements de ma propre démarche de mise en chanson de textes poétiques et prendre conscience de la pertinence de cette hypothèse de travail. Face à la multiplication d'ouvrages, revues et productions musicales consacrées à la poésie mise en chanson, il semblerait difficile de nier l'existence d'un véritable processus de canonisation de ce genre de la chanson à texte, non seulement en France. L'existence d'un double tremplin de la chanson française et italienne - le Prix Andrée Chedid et le Premio Lunezia - consacré à la mise en musique de textes poétiques, constitue également un élément important pour corroborer cette hypothèse. Le lecteur trouvera dans la section consacrée à la discographie quelques titres récents d'album où les musiciens chantent, chacun à leur façon, les poètes. Un dossier spécial de la revue Hexagone, conçu par David Desreumaux et Patrick Engel, pourra également offrir des compléments intéressants à ce sujet (Desreumaux/Engel 2018).

3 Il serait bien évidemment intéressant de poursuivre cette analyse en incluant également d'autres aires linguistiques, culturelles et géographiques, comme les organisatrices du colloque Chanter les poètes l'avaient suggéré, en citant le cas intéressant de l'Espagne (où le poète Miguel Hernández avait été mis en musique par Enrique Morente ou Carmen Linares) et des États-Unis (où le poète William Blake inspire le travail musical d'un Jim Morrison ou d'un Bob Dylan). Mais on pourrait ajouter le Canada (avec Leonard Cohen mettant en chanson Federico García Lorca) et plusieurs pays de l'Amérique latine où la fascination pour la poésie mise en chanson n'a pas été moins forte.

4 Voir en particulier le chapitre IV intitulé "Chanson et poésie, le malentendu " et la riche bibliographie l'accompagnant. Voir également les deux numéros de la revue Vox Popular consacrés à la question du rapport entre la poésie et la chanson (Conti/Tomatis 2016 ; Pruvost 2017).

5 C'est moi qui complète.

6 C'est ainsi que s'est exprimée l'Académie royale des sciences de Suède dans son communiqué officiel (cf. https://www.nobelprize.org/prizes/literature/2016/summary/ ; consultation 02.03.2019).

7 Car, comme l'affirme le philosophe Manlio Sgalambro (1997, 29), « le texte de chanson est un corps sonore, indissociable de la musique que le sort lui a donnée. Le destin de la chanson est différent de celui de la poésie et il est donc impossible de les comparer ». Traduction de Céline Pruvost $(2013,195)$.

8 Cf. "Trois hommes dans un salon : Brassens, Brel, Ferré ». Transcription de la rencontre disponible en ligne à l'adresse http://snoopairz.free.fr/ (consultation 02.03.2019).

9 http://snoopairz.free.fr/ (consultation 02.03.2019). 
10 Nous renvoyons à nouveau à l'excellent travail universitaire de Céline Pruvost pour une riche bibliographie concernant les diverses perspectives, à la fois théoriques et historiques, sur les rapports entre poésie, musique et chanson, y compris dans le cadre de la chanson d'auteur italienne (Pruvost 2013). À ce propos, voir en particulier la distinction faite par Stefano La Via entre poésie pour musique, poésie en musique et musique pour poésie (La Via 2006). En ce qui concerne, en particulier, la notion de chanson d'auteur italienne dans ses rapports avec la figure du « cantautore ", voir Deregibus (2009).

11 Pascal Pistone $(2018,31)$ parle à ce propos d'instinct arithmétique chez Ferré.

12 Ce sont les caractéristiques formelles qui permettent de définir la chanson comme genre ou type de musique, à savoir un «ensemble d'événements musicaux dont le déroulement est régi par des normes acceptées par une communauté donnée" (Fabbri 2003, 675).

13 La liste complète des cycles hamiltoniens « redondants `, c'est-à-dire décomposables en cycles de longueur plus petite, est disponible sur la page web du cours sur les modèles mathématiques dans la chanson que j'assure depuis 2017 dans la licence musiques actuelles de l'Université de Strasbourg. Voir à l'adresse : http://repmus.ircam.fr/moreno/chanson. La chanson est disponible en ligne, accompagnée d'une visualisation multimédia réalisée par Gilles Baroin, à l'adresse https:// www.youtube.com/watch?v=AB8By7ghTkU (consultation 02.03.2019).

14 La chanson est également disponible en ligne, toujours accompagnée d'une visualisation réalisée par Gilles Baroin, à la section « Music and Maths » de ma page web www.morenoandreatta.com (consultation 25.05.2020).

\section{Bibliographie}

Andreatta, Moreno : "Math'n pop : géométrie et symétrie au service de la chanson ». In : Tangente 51 (2013) : L'aventure mathématique, 92-97.

Andreatta, Moreno : "Modèles formels dans et pour la musique pop, le jazz et la chanson : introduction et perspectives futures ». In : Kapoula, Zoï / Lestocart, Louis-José / Allouche, JeanPaul (éds) : Esthétique \& Complexité II : Neurosciences, évolution, épistémologie, philosophie. Paris : éditions du CNRS, 2014, 69-88.

Andreatta, Moreno / Granger, Martin / Johnson, Tom / Villenave, Valentin : « Music, Mathematics and Language : Chronicles from the Oumupo Sandbox ». In : Kapoula, Zoï / Volle, Emmanuelle / Renoult, Julien / Andreatta, Moreno (éds) : Exploring Trandisciplinarity in Art and Science. Berlin : Springer, 2018, 255-274.

Aragon, Louis : "Léo Ferré et la mise en chanson ". Préface au disque de Léo Ferré Les Chansons d'Aragon, 1961. Texte disponible en ligne à l'adresse : http://www.jechantemagazine.com/Dossier_ Aragon/Dossier_Aragon/Entrees/2009/12/9_Leo_et_Louis.html (consultation 02.03.2019).

Bigo, Louis / Andreatta, Moreno : "A Geometrical Model for the Analysis of Pop Music ». In : Hascher, Xavier / Ayari, Mondher (éds) : La modélisation en analyse musicale. Numéro spécial de Sonus 35,1 (2014), 36-48. 
Conti, Jacopo / Tomatis, Jacopo (éds) : Poesia e canzone dalla Francia all'Europa. Vox Popular 1,1 (2016), http://www.voxpopular.it/vox-popular-11/ (consultation 02.03.2019)

Deregibus, Enrico : «Le radici culturali di Fabrizio De André ». In : Centro Studi Fabrizio De André (éd.) : Il suono e l'inchiostro. Cantautori, saggisti, poeti a confronto. Milan : Editions Chiarelettere, 2009, 18-27.

Desreumaux, David / Engel, Patrick (éds) : "Quand la littérature prend l'air. Chanson \& poésie ». In : Hexagone 14 (printemps 2018), 14-27.

Dylan, Bob : Discours à l'Académie suédoise. Paris : Fayard, 2017.

Fabbri, Franco : «La chanson ». In : Nattiez, Jean-Jacques et al. (éds) : Musiques. Une encyclopédie pour le XXI siècle. Vol. 1 : Musiques du XXe siècle. Arles/Paris : Actes Sud/Cité de la Musique, 2003, 674-702.

Ferré, Léo : "Aragon et la composition musicale ». Préface au disque de Léo Ferré Les Chansons d'Aragon, 1961. Texte disponible en ligne à l'adresse : http://www.jechantemagazine.com/Dossier_ Aragon/Dossier_Aragon/Entrees/2009/12/9_Leo_et_Louis.html (consultation 02.03.2019).

Hirschi, Stéphane : «Le malentendu des relations entre chanson et poésie ». In : Hirschi, Stéphane : Chanson, l'art de fixer l'air du temps : de Béranger à Mano Solo. Paris : Les Belles lettres/Presses universitaires de Valenciennes, 2008, 65-96.

La Via, Stefano : Poesia per musica e musica per poesia. Dai trovatori a Paolo Conte. Roma : Carocci, 2006 (avec CD-ROM).

Moustaki, Georges : La sagesse du faiseur de chanson. Paris : éditions Jean-Claude Béhar, 2011.

Pestel, Véronique : «Le charme d'Orphée ». In : Faites entrer l'Infini 54 (décembre 2012) : Aragon sous le regard de 25 écrivains et artistes d'aujourd'hui, 81-82. [L'article a été retiré du site de la revue, mais il est disponible sur : https://salaet.pagesperso-orange.fr/aragon/pages/feli/feli54/Pestel.pdf (consultation 02.03.2019).]

Pistone, Pascal : « ¿ L'Invitation au voyage > (Baudelaire, Ferré) : l'instinct arithmétique ». In : July, Joël / Pistone, Pascal (éds) : Ferré...vos papiers! Aix/Marseille : Presses universitaires de Provence, 2018, 31-44.

Pruvost, Céline : La chanson d'auteur dans la société italienne des années 1960 et 1970 : une étude cantologique et interculturelle. Thèse de doctorat. Sorbonne Universités 2013.

Pruvost, Céline (éd.) : Poésie et chanson de la France à l'Europe. Vox Popular 1,2 (2017), http://www. voxpopular.it/vox-popular-12/ (consultation 02.03.2019).

Sgalambro, Manlio : Teoria della canzone. Milano : Bompiani, 1997.

Vincenot, Matthias : Les mots et la note. Poésie et chanson : un cousinage compliqué. Paris : Editions de l'Amandier, 2014.

Vincenot, Matthias: Poésie et Chanson, stop aux a priori! 100 pages pour remettre les pendules à l'heure. Paris : Editions Fortuna, 2017. 


\section{Discographie}

Artistes variés : D'1 siècle à l'autre - mélodies françaises. Dièse Records DSR\#03, 2007 (CD).

Artistes variés : La bande des mots. Universal Music/Polydor 278 971-8, 2012 (CD).

Atlas, François : Fleurs du mal. Silène 198339, 2018 (33 tours).

Bruni, Carla : No Promises. Universal Music Division Barclay 811491, 2007 (CD).

Ferré, Léo : Baudelaire. Les fleurs du mal. Odéon OSX127, 1957 (33 tours).

Ferré, Léo : Les chansons d'Aragon. Barclay 80138, 1961 (33 tours).

Ferré, Léo : Léo Ferré chante Baudelaire. Barclay 80029, 1967 (33 tours).

Louis, Bertrand : Baudelaire. EPM Musique 6771150, 2018 (CD).

Modiano, Marie : Pauvre chanson. Nest \& Sound PVP12CD, 2018 (CD).

Pestel, Véronique : Caf' Conf Aragon. Jean-Claude Barens et FestiVal-de-Marne, 2013 (CD).

Populo, Barrio : Cris d'écrits. Carotte Production BP004, 2017 (CD). 